\title{
ABDOMINAL CUTANEOUS NERVE ENTRAPMENT SYNDROME (ACNES)
}

Markandu Thirukumar'; Thambawita H.R ${ }^{2}$

${ }^{1}$ Department of clinical sciences, FHCS, Eastern University

${ }^{2}$ Teaching Hospital Batticaloa

\section{Abstract}

Abdominal cutaneous nerve entrapment syndrome is caused by entrapment of an intercostal nerve in a fibrous ring in the rectus abdominis muscle and causes neuropathic pain. It remains an overlooked cause of chronic abdominal wall pain. Carnett's test is useful to make a diagnosis. An injection of local anaesthetic and corticosteroid combination relieves pain and it is both diagnostic and treatment. This review article describes its pathophysiology, clinical diagnosis and its management. The databases Medline, and Google Scholar were searched using the terms chronic abdominal pain in general, surgical and gynaecological practice. Database were merged and duplicates were removed. The aim of the review is to update the knowledge on this topic in day to day clinical practice

\section{Introduction}

The chronic abdominal pain gives anxiety and loss of work and economy to both to patients and health care system. Therefore, it demands several investigations and management modalities. The differential diagnoses of chronic abdominal pain are intra-abdominal disorders such as irritable bowel syndrome, spastic colon, and gastritis. When a correct diagnosis is not arrived, they are given a psychiatric diagnosis such as psychoneurosis, depression, anxiety, hysteria and malingering.

There is an under recognized and underappreciated cause of chronic abdominal pain, called abdominal cutaneous nerve entrapment syndrome (ACNES).
If a patient presents with chronic abdominal pain and no diagnosis is arrived, ACNES should be considered as a most probable diagnosis. $(1,2)$

\section{Epidemiology}

It is estimated that incidence of ACNES is 1 in 1800 . Further, it is seen in up to $30 \%$ of the patients with chronic abdominal pain who had negative results of prior diagnostic work up. (2)

The peak incidence of the condition is seen among the age group of 30-50 years and it is reported in $12 \%$ of pediatric outpatients with chronic abdominal pain.

\section{Pathophysiology of ACNES}

The anterior abdominal wall receives its sensory supply via the anterior and lateral cutaneous branches of the anterior rami of the 7 th -12 th thoracic nerves.

The infrasternal area is supplied by the T7; the umbilicus is by T10; the suprapubic are by $\mathrm{T} 12$ and L1 by the iliohypogastric and ilioinguinal nerve (Figure 1).

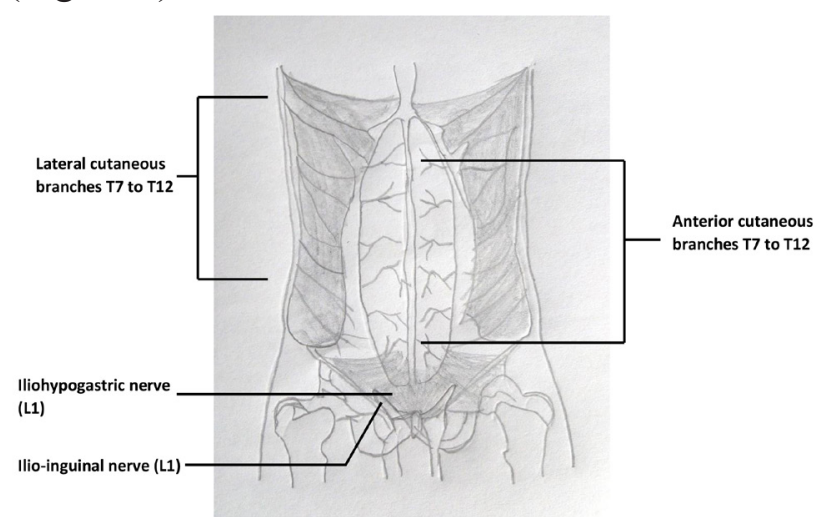

Figure 1: The anterior abdominal wall

Corresponding Author : Markandu Thirukumar, dr.thiru10@yahoo.com, iD https://orcid.org/0000-0001-8499-7175 
The cutaneous sensory nerves and vascular bundles lie in the plane between the internal oblique and transverses abdominis muscles [figure 2]. They supply the skin after passing towards the posterior wall of the rectus sheath and through the neurovascular channel in the rectus muscle.

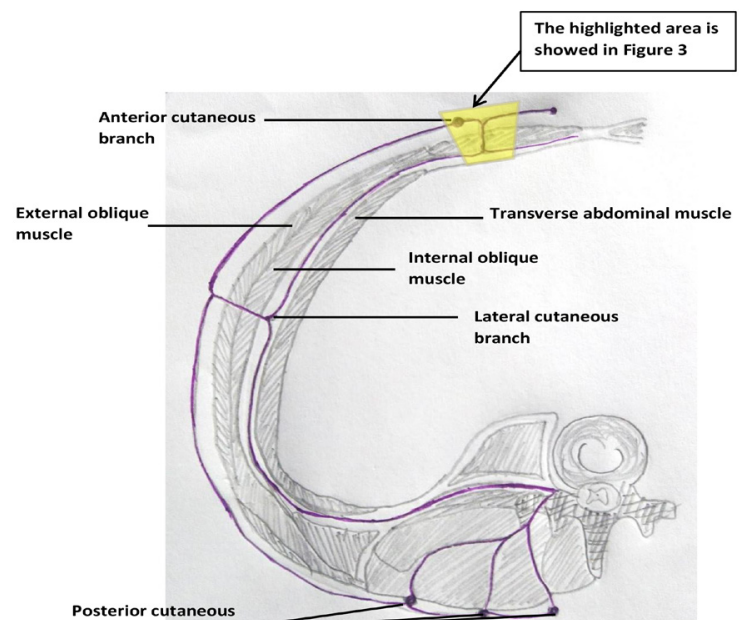

Figure 2: Anatomy of thoraco abdominal nerves.

These neurovascular channels freely mobile within a fibrous ring in the rectus muscle [figure 3]. Entrapment and mechanical irritation occur when they change the direction to enter a fibrous or osseo-fibrous tunnel or when passes over a fibrous or muscular band. (3)

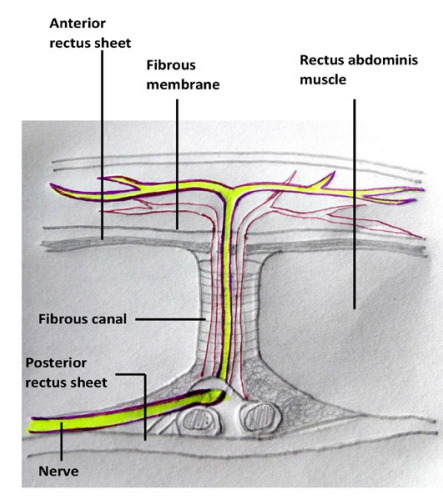

Figure 3: The course of the sensory nerves of the anterior abdominal wall.

Fibrous ring is the most susceptible for entrapment and a site of nerve compression and ischaemia which produces the symptoms of ACNES. Nerve traction and compression is also caused by rectus muscle contraction.

Localised swelling due to the irritation may directly injure the nerve or compromise the nerve's circulation. Valleix phenomenon explains that the tenderness of the main nerve trunk may be found proximally or distally to the affected portion. Proximal tenderness may result from vascular spasm or from unnatural traction on the nerve trunk against the point of entrapment. In ACNES, all these mechanisms can be at work. (4)

\section{Clinical presentation}

Patients often present with abdominal wall pain mainly at the right side lateral edge of the rectus abdominis muscle, but can be in multiple locations. Also the pain radiates to the affected dermatome.

It is sharply localized to a small $(<2 \mathrm{~cm})$ area of that is always felt in the same place and is usually dull or stabbing type. There are features of neuropathic pain such as retrograde radiation (Valleix phenomenon) due to entrapment neuropathy. The pain gets aggravated when the patient lies on the affected side or sits. Tight clothing, sneezing, coughing, laughing, and physical exercise are other aggravating factors. Even though patients often do not feel "sick," their quality of life can be impaired.

Pain is felt horizontally in the upper abdomen and more oblique in the lower abdomen due to the course of nerves responsible for ACNES in these regions. When the radiation occurs only with movement, it suggests the entrapment within the muscle. When the cutaneous nerve branches entrapped in scar tissue following abdominal surgeries, the direction of pain radiation shows the dermatomal distribution of the particular nerve entrapped.

There are recognized risk factors for ACNES; such as previous laparotomy and laparoscopic surgery and rectus muscle strengthening exercises. In addition, obesity, pregnancy and oral contraceptive use are also other risk factors for ACNES.

\section{Physical examination}

The physical examination is performed when the patient is in supine position and it is important to arrive at a diagnosis of ACNES. Pain is exactly localized with a fingertip at the linea semilunaris, i.e., the lateral border of the rectus abdominis muscle in most of the patients. 


\section{Carnett's test}

When pressing on the point of greatest pain with the finger tip, the pain gets worsened when the anterior abdominal wall is being contracted (Positive Carnett's test). But pain does not always become worse during the examination.

When the pain originates from the abdominal viscera, pain is less marked during the examination (Negative Carnett's test). Adequate voluntary contraction of the anterior abdominal musculature is essential for the proper examination.

Neurovascular channel is constricted when the rectus muscle contracts and worsens the symptoms of neuropathy. Abdominal hernias, abdominal wall haematomas, and rib tip syndrome also produce positive Carnett's sign on examination. (5)

Pressure over the nerve at the anterior openings in the rectus sheath will cause pain (Positive Hover sign).

Hypaesthesia, hyperalgesia, or allodynia around the area of pain also supports a diagnosis of ACNES and it has been reported by $75 \%$ of the patients with ACNES. (6)

The "pinch test" is useful if the origin of the site of the pain is not identified. This test is picking up the patient's skin with the subcutaneous fat between the thumb and index finger, first on one side of the midline of the abdomen and then on the other side. The patient will state whether one side hurts more than the other. Cotton and pinprick technique can be used to check for hypoesthesia or hyperesthesia around the pain site. (7)

\section{Management}

Health education about ACNES and the rectus muscle stretching exercises should be given to all patients. Though the efficacies of non-specific pharmacological therapies are unclear, heat or cold application, abdominal binders and transcutaneous electrical nerve stimulation are useful.

For instance, paracetamol, non-steroid antiinflammatory, anti-convulsant, anti-depressants, topical treatments such as 5\% lidocaine plasters, and capsaicin cream and opioids.

\section{Recommended Treatment for ACNES}

First line therapy is generally injection of local anaesthetic and corticosteroid combination which relieves pain and reduce the herniation of neurovascular bundle through the ring. A local injection of an anesthetic agent completely relieves the pain. The combine injection is the most commonly used one to treat ACNES. And it is both diagnostic and treatment.

0.5 to $1 \mathrm{ml}$ of $2 \%$ Lidocaine is used and the length of needle varies according to the thickness of subcutaneous tissue. Usually $21 \mathrm{G}$ or $22 \mathrm{G}$ is used. Spinal may be needed sometimes to reach the injection point. (8)

\section{Technique for inserting the needle}

There are many techniques to identify the landmark for injection. Palpation with fingers, nerve stimulator to identify the nerve and ultra sound guided injection. Ultrasound-guided local anaesthetic injection is increasingly recommended nowadays in the literature and it gives the median duration of pain relief of 12 weeks. (9)

There are palpable depressions on the lateral edge of the rectus muscle and this is the point of injection where the needle is introduced through skin, subcutaneous tissue, aponeurosis and to the fatty plug surrounds the neurovascular bundle when emerging from the fibrous channel.

When passing through the tissues, aponeurosis and the fatty plug produce resistant to the needle and the needle should not be inserted deeper than this level as this will further increase the pressure within the fibro muscular channel.

The tip of the needle should be placed in front of the fibrous ring just beneath the aponeurosis, and the examiner should make sure the position of the needle before the injection by pulling out the needle into the subcutaneous tissue to insert again.

The injection is best given in patient standing and Jaffna Medical Journal 
bearing down position. But can be given in lying position if the patient is comfortable.

Position the needle with middle finger of one hand in the aponeurotic opening and use the other hand for cleaning the area with alcohol and inserting the needle above the tip of the finger. The other hand should not be taken off until the needle is being situated in correct position and use the same hand to stabilize the needle while injecting the drugs. Patient should be explained not to breathe during the injection.

Neuromodulation using pulse radiofrequency lesion has been attempted prolong the pain relief. There is an inflicting a second nerve injury resulting with these injection procedures a small but significant risk. Surgical options are available for ACNES. Suggest surgical neurectomy for ACNES, but the long-term outcome is yet to be known. $(10,11)$

\section{References}

1. Suleiman S, Johnston DE. The abdominal wall: an overlooked source of pain. Am Fam Physician 2001 Aug 1;64(3):431-8.

2. ApplegateWV. Abdominal cutaneous nerve entrapment syndrome (ACNES): a commonly overlooked cause of abdominal pain. Perm J 2002; 6: 20-7.

3. Kopell HP, ThompsonWA. Peripheral Entrapment Neuropathies. Malabar, FL:Robert E. Kreiger Publishing, 1976; 1-7, 85-8.

4. Srinivasan R, Greenbaum DS. Chronic abdominal wall pain: a frequently overlooked problem. Practical approach to diagnosis and management. Am J Gastroenterol 2002; 97: 824-30.
5. Hershfield NB. The abdominal wall. A frequently overlooked source of abdominal pain. J Clin Gastroenterol 1992; 14: 199 202.

6. Boelens OBA, Scheltinga MR, Houterman $S$, Roumen RM. Randomised clinical trial of trigger point infiltration with lidocaine to diagnose anterior cutaneous nerve entrapment syndrome. Br J Surg 2013; 100:217-21.

7. KnockaertDC, Boonen AL, Bruyninckx FL, Bobbaers HJ.Electromyographic findings in ilioinguinal-iliohypogastric nerve

8. Koop H, Koprdova S, Schürmann C: Chronic abdominal wall pain-a poorly recognized clinical problem. Dtsch Arztebl Int 2016; 113: 51-7. DOI: 10.3238/ arztebl.2016.0051.

9. Kanakarajan S, High K, Nagaraja R. Chronic abdominal wall pain and ultrasound-guided abdominal cutaneous nerve infiltration: a case series.

Pain Med 2011; 12: 382-6.

10. Boelens OBA, van Assen T, Houterman $S$, Scheltinga MR, Roumen RM. A doubleblind, randomized, controlled trial on surgery for chronic abdominal pain due to anterior.

11. Zganjer M, Bojic D, Bumci I. Surgery for abdominal wall pain caused by cutaneous nerve entrapment in children-a single institution experience in the last 5 years. Iran Red Crescent Med J 2013; 15: 15760. 